\title{
Hyperemic contrast velocity assessment improves accuracy of the image-based fractional flow reserve calculation
}

\author{
Balázs Tar ${ }^{1,2}$, Csaba Jenei ${ }^{2,3}$, Áron Üveges ${ }^{1,2}$, Gábor Tamás Szabóo ${ }^{2,3}$, András Ágoston ${ }^{1}$, \\ Csaba András Dézsi ${ }^{4}$, András Komócsi ${ }^{5}$, Dániel Czuriga ${ }^{2,3}$, Attila Juhász ${ }^{6}$, Zsolt Kőszegi1 ${ }^{1,2,3}$ \\ ${ }^{1} 3^{\text {rd }}$ Department of Internal Medicine, Szabolcs - Szatmár - Bereg County Hospitals \\ and University Teaching Hospital, Nyíregyháza, Hungary \\ ${ }^{2}$ Kálmán Laki Doctoral School of Biomedical and Clinical Sciences, \\ University of Debrecen, Hungary
}

${ }^{3}$ Division of Cardiology, Department of Cardiology, Faculty of Medicine, University of Debrecen, Hungary

${ }^{4}$ Aladár Petz County Teaching Hospital, Győr, Hungary

${ }^{5}$ Heart Institute, Medical School, University of Pécs, Hungary

${ }^{6} \mathrm{GE}$ Healthcare Limited, Pharmaceutical Diagnostics, Pollards Wood, United Kingdom

Collet et al. [1] published a meta-analysis about the diagnostic performance of angiography-derived (image-based) fractional flow reserve (FFR). They concluded that the angiography-derived FFR was suitable for detecting hemodynamically significant lesions with good accuracy compared with pressure wire measured FFR as a reference. Thirteen studies were included in this systematic review; hyperemia was induced in three studies [2-4] and the comparison of the results of FFR values from hyperemic and baseline velocity data was published only in the FAVOR pilot study (FAVOR1) [3].

In our previous study, on a less invasive FFR measurement, we proposed a new simplified model developed for the estimation of FFR [2]. It was found that this method was suitable for characterizing every single intermediate stenosis with an especially good positive predictive value; furthermore, the calculations only require a simple MS Excel sheet for the input of the hyperemic flow velocity values and the data of the three-dimensional coronary reconstruction. During this previous study, real patient specific flow information was applied in every case using the hyperemic frame count data during i.c. adenosine challenge. The translesional pressure gradients were calcu- lated according to simple fluid dynamic equations as published in an earlier paper by our group [5].

The purpose of this present study was to perform a retrospective analysis on the cases of the previous study [2] to assess the accuracy of FFR calculations using the fixed hyperemic contrast flow, the resting frame count, and the hyperemic frame count data.

Fifty patients with intermediate severity epicardial coronary artery disease (40-70\% diameter stenosis) were re-evaluated. The details of the measurements were published in Tu et al. [4]. Diagnostic angiographic images were recorded at 15 frame/sec both in resting and hyperemic conditions. Low- or iso-osmolar contrast material (CM) (iopamidol [Scanlux] or iodixanol [Visipaque]) was injected in $5 \mathrm{~mL}$ fractions with a speed of $3 \mathrm{~mL} / \mathrm{s}$ using a dedicated contrast pump (Fig. 1).

The calculations were performed using three different velocity values:

- fixed $\mathrm{FFR}_{\mathrm{sim}}$ : calculated from the fixed hyperemic velocity $(35 \mathrm{~cm} / \mathrm{s})$;

- rest $\mathrm{FFR}_{\text {sim }}$ : calculated using the non-hyperemic frame count data to extrapolate the hyperemic velocity. For extrapolation the quadratic relation between the baseline contrast flow velocity $(\mathrm{CFV})$ and the hyperemic flow velo-

Address for correspondence: Zsolt Kőszegi, MD, PhD, University of Debrecen Medical Center, Móricz. Zs. krt. 22 , 4032 Debrecen, Hungary, tel: 36-30-2589442, e-mail: koszegi@med.unideb.hu

Received: 29.07.2020 Accepted: 13.10 .2020

This article is available in open access under Creative Common Attribution-Non-Commercial-No Derivatives 4.0 International (CC BY-NC-ND 4.0) license, allowing to download articles and share them with others as long as they credit the authors and the publisher, but without permission to change them in any way or use them commercially. 


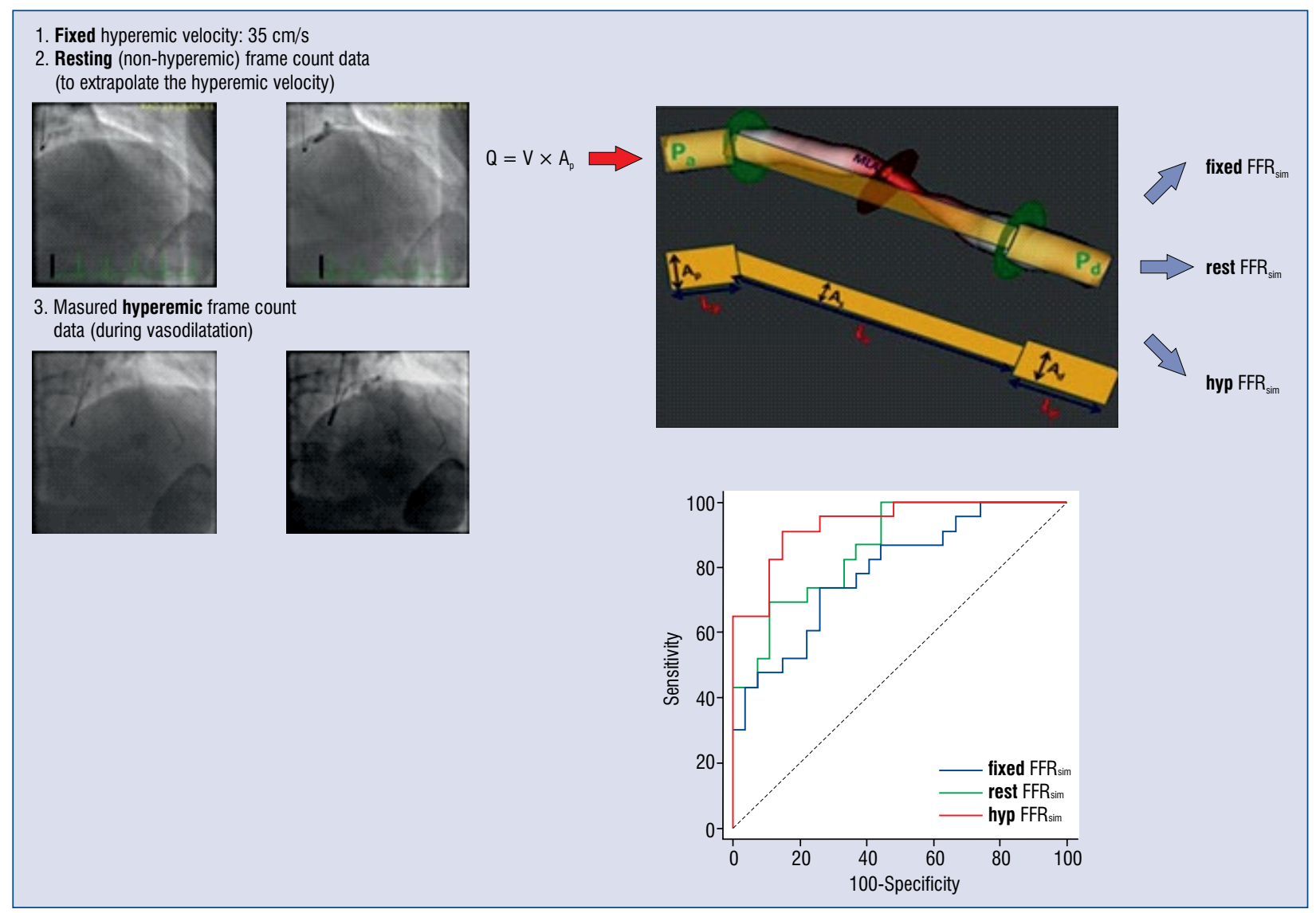

Figure 1. Different calculations of the $\mathrm{FFR}_{\text {sim }}$ and the comparison of receiver operating characteristic (ROC) curves. Flow velocities for pressure drop calculations were applied: 1 . Fixed hyperemic velocity: $35 \mathrm{~cm} / \mathrm{s} ; 2$. Resting (non-hyperemic) frame count data to extrapolate the hyperemic velocity according to a database: $\mathrm{HFV}=\mathrm{a}_{0}+\mathrm{a}_{1} \times \mathrm{CFV}+$ $+a_{2} X_{C F V}\left(a_{0}=0.10 ; a_{1}=1.55 ; a_{2}=-0.93\right)$, where HFV is hyperemic flow velocity, CFV is a resting contrast flow velocity) [5]; 3. Measured hyperemic frame count data (during vasodilatation). Comparison of ROC curves: The hyp FFR sim showed the strongest prediction of the measured FFR values. The area under the curve (AUC) for hyp FFR sim $_{\text {: }} 0.936$ (95\% Cl 0.828-0.985), for rest $\mathrm{FFR}_{\text {sim }} 0.862(95 \% \mathrm{Cl} 0.734-0.943)$ and for fixed $\mathrm{FFR}_{\text {sim }}: 0.791$ (95\% Cl 0.652-0.893); $p=0.011$ between hyp FFR sim $_{\text {and }}$ and rest FFR $R_{\text {sim }} ; p=0.005$ between hyp FFR $R_{\text {sim }}$ and fixed $F F R_{\text {sim }}$.

city (HFV) was applied and was published in the FAVOR1 study [5]: HFV $=\mathrm{a} 0+\mathrm{a} 1 \mathrm{xCFV}$ $+\mathrm{a} 2 \mathrm{xCFV}^{2}$, where $\mathrm{a} 0=0.10$, a $1=1.55$ and a2 = -0.93;

- hyp $\mathrm{FFR}_{\text {sim }}$ : values from hyperemic frame count assessment [4].

The area under the curve (AUC) by receiver operating characteristic (ROC) analysis was used to assess the diagnostic power of $\mathrm{FFR}_{\text {sim }}$.

The diagnostic powers of different computations of the $\mathrm{FFR}_{\mathrm{sim}}$ was assessed comparing the results to standard invasive FFR measurements $\left(\mathrm{FFR}_{\text {meas }}\right)$. Based on ROC curve analysis for predicting the abnormal FFR of $\leq 0.80$ the AUC were significantly higher for the hyperemia-based parameters than for those calculated with the resting frame counts: 0.936 (95\% confidence interval
[CI] 0.828-0.985) for hyp $\mathrm{FFR}_{\text {sim }}, 0.862(95 \% \mathrm{CI}$ $0.734-0.943)$ for rest $\mathrm{FFR}_{\text {sim }}$, and $0.791(95 \% \mathrm{CI}$ $0.652-0.893)$ for fixed $\mathrm{FFR}_{\text {sim }}(\mathrm{p}=0.011$ between hyp $\mathrm{FFR}_{\text {sim }}$ and rest $\mathrm{FFR}_{\text {sim }} ; \mathrm{p}=0.005$ between hyp FFR $\mathrm{Fim}_{\text {si }}$ and fixed FFR $\mathrm{Fim}_{\text {sim }}$; Fig. 1).

Despite the potential confounding factors of the Thrombolysis in Myocardial Infarction (TIMI) frame count measurement, it was proven that its reproducibility is acceptable for clinical purposes [6]. However, the heart rate and the phase of the cardiac cycle in which the dye is injected have been reported to exert significant effects on the TIMI frame count determination [7].

The effect of timing of the injection on the produced contrast speed in the different phases of the cardiac cycle is evident: there are significantly different coronary flow velocities during the 
phases of the heart cycle - the diastolic velocity is usually twice as high as the systolic velocity in the left coronary artery. Theoretically it would be possible to correct the assessed frame count to the assumed average blood flow by relating the determined frame count to the measuring interval of the cardiac cycle by an appropriate algorithm to be developed in the future.

In contrast to the FAVOR1 study [3], the work herein, demonstrates that the hyperemic velocity assessment is superior for patient specific calculation of the hyperemic pressure gradient i.e. the FFR. The diagnostic performance of hyperemic frame count determination is significantly higher according to the ROC analysis than the resting frame count-based calculations. The differing results of the FAVOR1 study can be explained by the different patient populations in the two studies. While in the FAVOR1 study only $31.5 \%$ of the patients had prior myocardial infarction, in the present patient population prior myocardial infarction had occurred in $51.6 \%$ of patients [3, 4]. This implies that in our population, the distal microvasculature could be damaged more frequently with consequent impairment of the vasodilator capacity [8].

In our opinion, the calculation of the extrapolated image based FFR without vasodilation could only be acceptable if the results are unequivocal - either below 0.70 or above 0.90 . However, in the range of FFR $0.70-0.90$, vasodilator challenge is highly reasonable, especially if the presence of microvascular disease is suspected. Currently there is no other viable approach to increase the accuracy of image-based FFR without assessing patient specific hyperemic velocity.

Although image-based FFR calculation without hyperemia seemed to be accurate enough for clinical purposes, the vasodilator challenge is highly reasonable for personalized accuracy of the investigation, especially when the presence of microvascular disease is suspected. An exact heart cycle correction for the hyperemic velocity assessment could further improve the accuracy of image-based FFR determination.
Conflict of interest: Dániel Czuriga was supported by the János Bolyai Research Scholarship of the Hungarian Academy of Sciences (BO/00523/16/5); Attila Juhász was a full-time employee of GE Healthcare Ltd. but has no conflict to disclose in relation to this paper; Zsolt Köszegi received a research grant from GE Healthcare Ltd. Other authors have no conflict of interest to declare.

\section{References}

1. Collet C, Onuma Y, Sonck J, et al. Diagnostic performance of angiography-derived fractional flow reserve: a systematic review and Bayesian meta-analysis. Eur Heart J. 2018; 39(35): 3314-3321, doi: 10.1093/eurheartj/ehy445, indexed in Pubmed: 30137305.

2. Tar B, Jenei C, Dezsi CA, et al. Less invasive fractional flow reserve measurement from 3-dimensional quantitative coronary angiography and classic fluid dynamic equations. EuroIntervention. 2018; 14(8): 942-950, doi: 10.4244/EIJ-D-17-00859, indexed in Pubmed: 29488883.

3. Tu S, Westra J, Yang J, et al. FAVOR Pilot Trial Study Group. Diagnostic Accuracy of Fast Computational Approaches to Derive Fractional Flow Reserve From Diagnostic Coronary Angiography: The International Multicenter FAVOR Pilot Study. JACC Cardiovasc Interv. 2016; 9(19): 2024-2035, doi: 10.1016/j. jcin.2016.07.013, indexed in Pubmed: 27712739.

4. Tu S, Barbato E, Köszegi $Z$, et al. Fractional flow reserve calculation from 3-dimensional quantitative coronary angiography and TIMI frame count: a fast computer model to quantify the functional significance of moderately obstructed coronary arteries. JACC Cardiovasc Interv. 2014; 7(7): 768-777, doi: 10.1016/j. jcin.2014.03.004, indexed in Pubmed: 25060020.

5. Koszegi Z, Tar B, Ember S, et al. Calculation the translesiona pressure gradients on coronary stenosis by combining three dimensiona coronary angiography parameters with frame count data. Computing in Cardiology. 2011; 38: 729-32.

6. Gibson CM, Cannon CP, Daley WL, et al. TIMI frame count: a quantitative method of assessing coronary artery flow. Circulation. 1996; 93(5): 879-888, doi: 10.1161/01.cir.93.5.879, indexed in Pubmed: 8598078.

7. Abaci A, Oguzhan A, Eryol NK, et al. Effect of potential confounding factors on the thrombolysis in myocardial infarction (TIMI) trial frame count and its reproducibility. Circulation. 1999; 100(22): 2219-2223, doi: 10.1161/01.cir.100.22.2219, indexed in Pubmed: 10577994.

8. Mejía-Rentería H, Lee JM, Lauri F, et al. Influence of microcirculatory dysfunction on angiography-based functional assessment of coronary stenoses. JACC Cardiovasc Interv. 2018; 11(8): 741-753, doi: 10.1016/j.jcin.2018.02.014, indexed in Pubmed: 29673505. 